\title{
Etno-ornitología del género Sicalis en Mollendo, Arequipa
}

\author{
Ethno ornithology of genus Sicalis in Mollendo, Arequipa \\ ${ }^{1, a}$ José Pizarro Neyra
}

NOTA CIENTÍFICA
${ }^{1}$ Asociación para el Desarrollo de
las Ciencias Biológicas en el Perú.
Tacna, Perú.
E-mail:
josepizarroneyra@gmail.com

aRCID: 0000-0002-4393-6277

Palabras clave: chollonco, pichí, Sicalis, etno-ornitología, Mollendo

Keywords: chollonco, pichí, Sicalis, etno-ornithology, Mollendo

Información adicional

Presentado: 30/04/2021

Aprobado: 20/05/2021

\section{RESUMEN}

Algunos aspectos etno-ornitológicos de Sicalis luteola Sparrman 1789 y Sicalis raimondii Taczanowski 1874 se explican en esta nota. El trabajo de campo se llevó a cabo en Mollendo, distrito de la provincia de Islay, departamento de Arequipa durante diciembre del 2020 y enero del 2021. La metodología utilizada fue la entrevista a informantes clave, quienes brindaron información sobre captura, cría y competencia en concursos a nivel local. Los resultados indican que los criadores de aves conocen bien su hábitat. Además, la información de los participantes sobre la morfología y vocalización de las aves coincide con la literatura; sin embargo, es impreciso en el tamaño de $S$. raimondii. De acuerdo con los entrevistados, $S$. raimondii se denomina "chollonco", mientras que $S$. luteola se conoce como "pichí". Otro dato relevante es que la captura de aves canoras silvestres en Mollendo se remonta al menos a la primera década del siglo XX y algunos pobladores la consideran una tradición local.

ABSTRACT
This note explains some ethnoornithological aspects of Sicalis luteola
Sparrman 1789 and Sicalis raimondii Taczanowski 1874. The field work took
place in Mollendo, district of the province of Islay, Department of Arequipa
during December 2020 and January 2021. The methodology used was the
interview with key informants, who provided information on the capture,
breeding and competition of birds in competitions at the local level. The results
indicate that bird breeders know the bird's habitat very well. In addition, the
information of the participants on the morphology and vocalization of the
birds coincides with the literature, but fail in the size of S. raimondii. The
participants named S. raimondii as "chollonco" while S. luteola is known as
"pichi'. Another relevant fact is that the capture of wild songbirds in Mollendo
began at least from the first decade of the 20th century and today it is
considered a local tradition by some inhabitants.




\section{INTRODUCCIÓN}

Las especies de aves canoras silvestres capturadas y exportadas en mayor número en el Perú durante los últimos cuarenta años pertenecen a los géneros Sicalis y Spinus (Ministerio de Agricultura, 1981; 1991; INRENA, 1996; Daut et al., 2015), es decir, se trata de los llamados chirigüies o triles además de jilgueros, respectivamente. Desde la perspectiva de la etno-ornitología, esto indica que existe una interacción importante entre este tipo de aves canoras silvestres y el hombre en el Perú. De acuerdo a Farias \& Alves (2007), la etno-ornitología se define como el conjunto de estudios en el que buscamos comprender las relaciones cognitivas, conductuales y simbólicas entre la especie humana y las aves. Sin embargo, el conocimiento tradicional acerca de las aves se caracteriza por ser holístico, no sistemático y contextual, así como desprovisto de una visión global de la biología como se cultiva en el mundo occidental (Gosler et al., 2010), por lo que cada especie o etno-especie de ave será percibida de distinta forma por cada grupo humano. Ello amerita un estudio de cada caso en etno-ornitología, lo que conlleva a reunir saberes sobre determinado tipo de ave que vive en un hábitat específico. De esta forma, Alves et al. (2017) justifica que un enfoque etno-zoológico puede ayudar a guiar programas que apuntan a la explotación sostenible y conservación de las especies, debido a que es imperativo comprender el contexto de explotación e interacción entre humanos y los recursos naturales para el desarrollo e implementación de medidas de conservación.

El caso de las especies del género Sicalis sujetas a captura en el distrito de Mollendo es importante debido a que allí se captura y se crían al menos dos especies: Sicalis luteola Sparrman 1789 y Sicalis raimondii Taczanowski 1874. En la provincia de Islay, S. luteola figura como parte del inventario de avifauna del Santuario Nacional Lagunas de Mejía (SERNANP, 2016). De acuerdo a Luque et al. (2019), se pueden observar cinco especies del género Sicalis en la región Arequipa, pero solo S. raimondii y $S$. luteola se consideran residentes en la costa. Estas dos especies han sido comercializadas en Arequipa como aves de jaula (Ortiz, 2010).

La presente investigación se realizó en la ciudad de Mollendo $\left(-17^{\circ} 01^{\prime} 00^{\prime \prime},-72^{\circ} 01^{\prime} 00^{\prime \prime}\right)$ entre diciembre de 2020 y enero de 2021. Mollendo es una ciudad con una población estimada al año 2020 de 26 453 habitantes (INEI, 2020), está localizada en la provincia de Islay, en el departamento de Arequipa. Mollendo se encuentra situada en el litoral y está rodeada de quebradas que en su límite superior albergan la formación vegetal de Lomas, la cual es endémica de Perú y el norte de Chile (Rundel et al., 1991).

\section{MATERIALES Y MÉTODOS}

Se empleó el método de investigación de entrevista a informantes clave (Albuquerque et al., 2014), es decir, obtención de información proveniente de personas poseedoras de conocimiento sobre las especies de aves mencionadas. Para ello, se seleccionaron personas bajo los siguientes criterios: que fueran ganadores del concurso de cholloncos, miembros de la Asociación de criadores y protectores del chollonco "El Relincho" o que tuvieran información histórica referente a la práctica de la actividad de captura de aves silvestres en el lugar. En diciembre del 2021, se tomó contacto con el presidente de la asociación y se logró presenciar el Concurso de Chollonco, el cual se realizó el 2 de enero del 2021 en Mollendo, auspiciado por una empresa privada de la localidad. En esta competencia solo participan ejemplares de $S$. raimondii (Figura $1)$. 


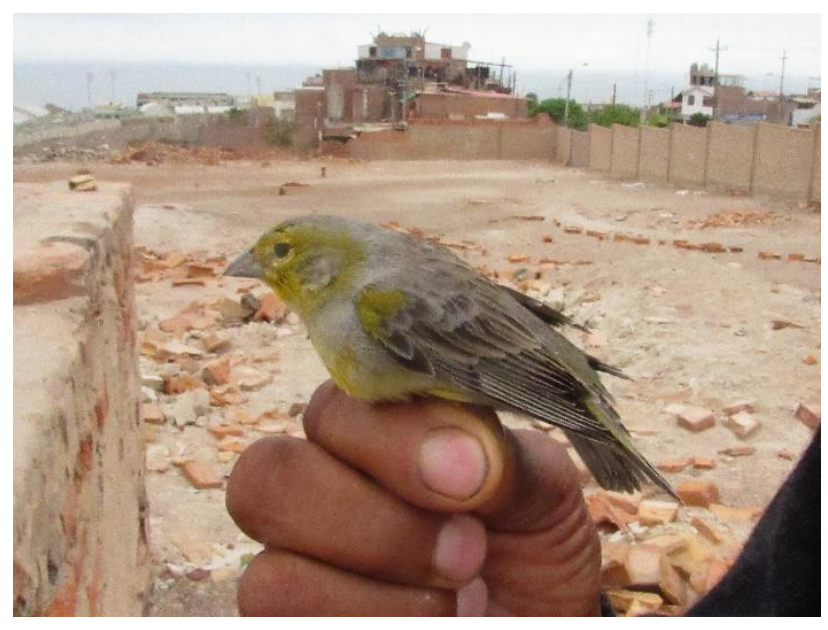

Figura 1. Ejemplar de Sicalis raimondii "chollonco"

Asistieron 118 concursantes al evento, no todos miembros de la asociación mencionada. Ese día se entrevistaron y filmaron con consentimiento verbal previo a 12 informantes clave, todos hombres, con edades entre 30 y 80 años y codificados del 1 al 12 con la finalidad de conservar su identidad en reserva. La información se guarda en el archivo de la Asociación para el Desarrollo de las Ciencias Biológicas en el Perú (ADCBP) de Tacna. Se entrevistaron a los participantes acerca de aspectos de captura, crianza y su valor como ave canora.

\section{RESULTADOS}

Los resultados se resumen en la Tabla 1, en donde se exhiben datos verbales transcritos de las grabaciones que son más frecuentes, indicando el número codificado del participante que brindó la información entre paréntesis. En algunos aspectos hubo consenso entre los entrevistados; por ello, no hay necesidad de señalarlo con la codificación anterior.

\section{Tabla 1}

Resumen de información acerca de aves del género Sicalis capturadas en Mollendo.

\begin{tabular}{|c|c|c|}
\hline \multirow[b]{2}{*}{ Características } & \multicolumn{2}{|c|}{ Aves del género Sicalis } \\
\hline & S. luteola & S. raimondii \\
\hline Nombre popular* & "pichí" & "chollonco" \\
\hline Origen del nombre & Onomatopéyico $(1,2)$ & Quechua $(10,12)$ \\
\hline $\begin{array}{l}\text { Diferencias entre "pichí" } \\
\text { y "chollonco" }\end{array}$ & $\begin{array}{l}\text {-Más grande que el chollonco } \\
\text {-El macho con flancos amarillos } \\
\text { y pecho blanco con manchitas } \\
\text { negras (3) }\end{array}$ & $\begin{array}{l}\text {-Mide como } 10 \mathrm{~cm}(1,2) \\
\text {-El macho con nuca, manto y } \\
\text { flancos grises. Pecho amarillo } \\
\text { (8) }\end{array}$ \\
\hline Vocalización* & $\begin{array}{l}\text { "pichí-pichí" }(1,2), \\
\text { "canta como el canario".(2,11) }\end{array}$ & $\begin{array}{l}\text { Al menos trece tipos de } \\
\text { llamadas } \\
\text { denominadas } \\
\text { diferentes } \\
\text { Algunas son conocidas como: } \\
\text { "chor", "ji-ji", "pito", } \\
\text { "campanilla", "carretilla", "cle- } \\
\text { cle", "grillo", "loro", } \\
\text { "caballitos", "ger-ger", "yu- } \\
\text { yu"y "chó-chó". }\end{array}$ \\
\hline
\end{tabular}




\begin{tabular}{|c|c|c|}
\hline \multirow[b]{2}{*}{ Características } & \multicolumn{2}{|c|}{ Aves del género Sicalis } \\
\hline & S. luteola & S. raimondii \\
\hline $\begin{array}{l}\text { Antigüedad de la } \\
\text { actividad }\end{array}$ & Desde el siglo $X X .(1,6,12)$ & $\begin{array}{l}\text { "Mis abuelos criaban } \\
\text { chollonco en 1914" (5) } \\
\text { "comienzos de S. XX" }(6,12) \text {, } \\
\text { "más de un siglo" (2). } \\
\text { "100 años" }(7,9)\end{array}$ \\
\hline Lugar de captura* & Valle de Tambo, Mejía. $(5,12)$ & $\begin{array}{l}\text { Lomas de la provincia de Islay } \\
\text { principalmente }\end{array}$ \\
\hline Época de captura* & Todo el año $(5,12)$ & $\begin{array}{l}\text { Setiembre a marzo del año } \\
\text { siguiente. }\end{array}$ \\
\hline Hábitat* & $\begin{array}{l}\text { Arbustos, zonas agrícolas, } \\
\text { totorales. }(5,12)\end{array}$ & $\begin{array}{l}\text { Anida en el interior de grietas } \\
\text { rocosas cerca de afloramientos } \\
\text { de agua o "aguadas". }\end{array}$ \\
\hline Etnoveterinaria & $\begin{array}{l}\text { Uso de medicina veterinaria } \\
\text { occidental }(2,12)\end{array}$ & $\begin{array}{l}\text { Uso de medicina occidental } \\
(1,2,5,12) \\
\text { Uso de plantas medicinales } \\
\text { provenientes de Lomas }(1,3,4, \\
9,10,11)\end{array}$ \\
\hline Valor cultural* & Ave de jaula & $\begin{array}{l}\text { Ave de jaula, tradición propia } \\
\text { de Mollendo, ave de } \\
\text { competencia para concursos de } \\
\text { "golpeo". }\end{array}$ \\
\hline Particularidad* & Solo se capturan machos & $\begin{array}{l}\text { Solo se capturan machos } \\
\text { debido a que solo estos } \\
\text { producen llamadas nupciales o } \\
\text { "golpeos" }\end{array}$ \\
\hline
\end{tabular}

Fuente: entrevistas a informantes clave. Los números entre paréntesis corresponden a los informantes clave citados. *Hubo consenso entre los participantes en las respuestas.

En cuanto al nombre popular de S. luteola, de acuerdo con De la Peña (2011), en Argentina se le conoce como "chollonco"; sin embargo, Pulido (1988) menciona que es llamado "chirigüe" en el Perú. Los entrevistados sostienen que su nombre local alude a su canto: "pichí-pichí" (Figura 2). Aunque el origen del nombre local de S. raimondii "chollonco" es atribuido al idioma quechua, no se menciona su significado. Sin embargo, este vocablo podría corresponder al término "Cchislluncaa", hallado en el Vocabulario de la lengua Aymara de Ludovico Bertonio, y que significa "paxarito negro y amarillo de estos que cantan" (Bertonio, 1612 [2006]).

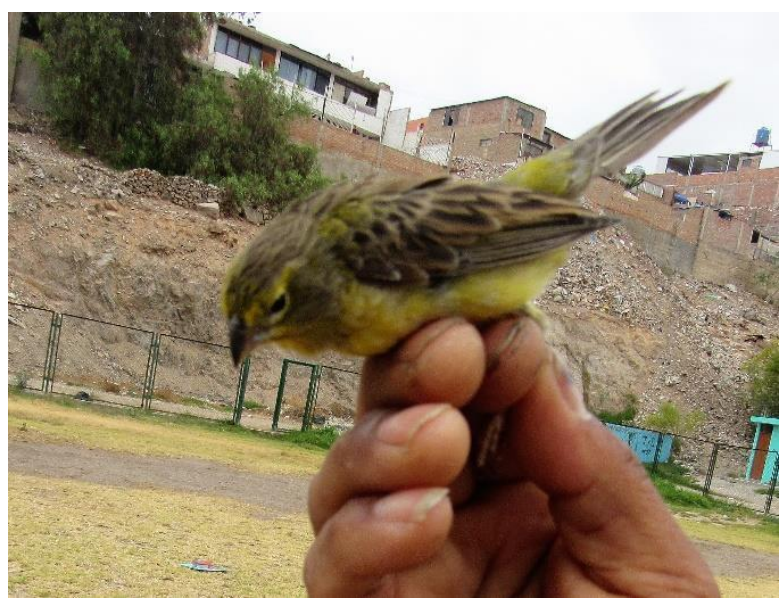

Figura 2. Ejemplar de Sicalis luteola "pichí" 
Por otro lado, el hecho de que algunos criadores capturen al "chollonco" en las lomas y utilicen plantas medicinales de uso etno-veterinario provenientes de ese mismo lugar para curar a las aves implica que han logrado acumular saberes sobre el dominio geográfico y hábitat de S. raimondii. Esto resulta ser una característica de los grupos humanos que interaccionan con aves, debido a que saber acerca de las aves requiere conocimiento de la tierra y otras criaturas porque todos están interconectados (Sault, 2010).

\section{DISCUSIÓN Y CONCLUSIONES}

En cuanto a las características de las aves expresadas por los participantes acerca de vocalización, morfología externa, hábitat y distribución geográfica, estas coinciden con la literatura (Hughes, 1991; Schulenberg et al., 2007; Ortiz, 2017). Sin embargo, el tamaño de S. raimondii expresado por la mayoría de participantes es más pequeño que el mencionado por Schulenberg et al. (2007).

El hecho de que solo se capturan machos implica que no se busca que el ave se reproduzca en cautiverio y que los criadores de Mollendo necesitan volver al campo para proveerse de más animales para criar, lo que les permite estar en contacto con la naturaleza permanentemente. Lo anterior ha influido para que algunos criadores desarrollen un conocimiento local acerca de detalles del hábitat, uso etno-veterinario de plantas, así como de las diferencias de vocalización de las aves, como se aprecia en la Tabla 1. Al visitar la casa de algunos criadores se apreció que se crían tanto ejemplares de "pichí" como de "chollonco", siendo estos últimos más numerosos (Figura 3).

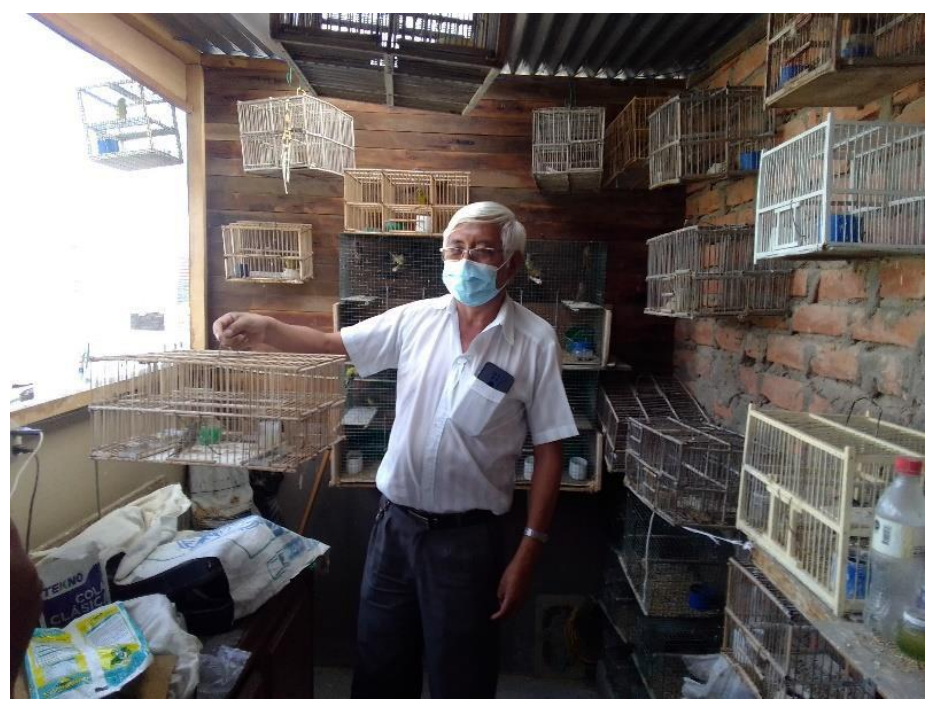

Figura 3. Criador de "chollonco" con sus aves en Mollendo.

Aunque existe un sesgo de género en la práctica de esta actividad, esta se presentaría a favor de los hombres en las tareas de captura y durante la realización del concurso de aves (Figura 4), sin embargo, se ha podido constatar que en la crianza de estas dos aves no habría asimetría en la participación por género. 


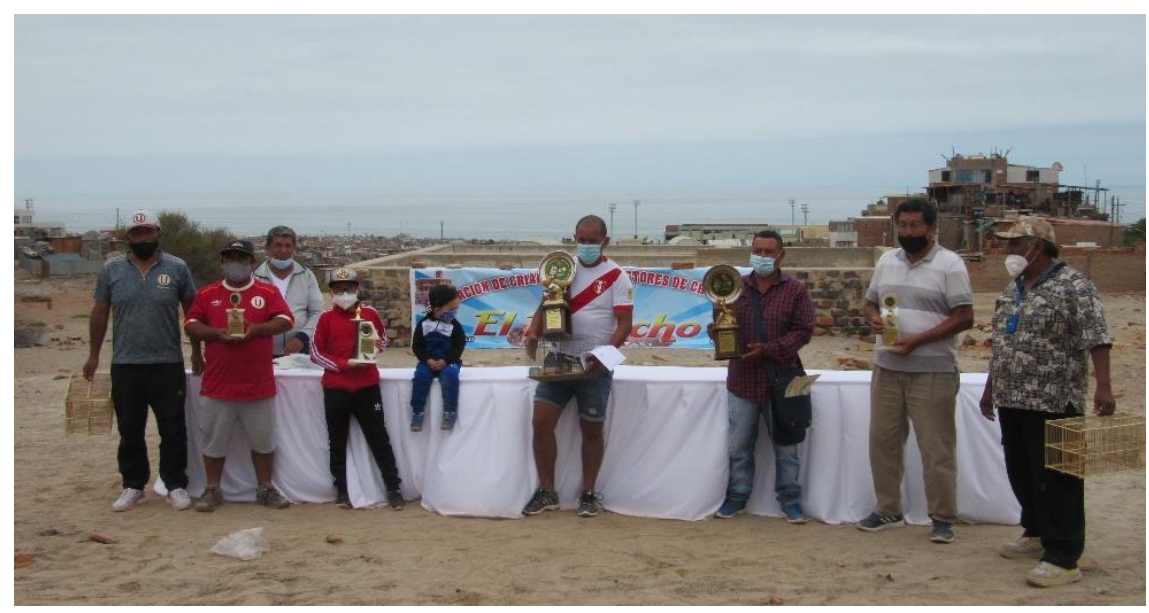

Figura 4. Ganadores del concurso de canto de "chollonco" 2021.

Finalmente, aunque la captura, cría y competencia de estas especies de aves no está reconocida oficialmente por el Ministerio de Cultura como una tradición local de importancia en el Perú, hay evidencias que indican que es una actividad tradicional de semi-domesticación de aves que se practica al menos desde la primera década del siglo XX (Chávez, 2019).

\section{AGRADECIMIENTOS}

A los miembros de la Asociación de criadores y protectores de Chollonco "El Relincho" de Mollendo, en especial al Prof. Efraín Minaya y al Sr. Arturo Pastor por su valiosa colaboración para el presente trabajo.

\section{REFERENCIAS}

Albuquerque, U. P., Lucena, R. F. P., Neto, \& E. M. F. L. (2014). Selection of Research Participants. In: Albuquerque U.P., Cruz da Cunha L.V.F., Lucena R.F.P., Alves R.R., editors. Methods and Techniques in Ethnobiology and Ethnoecology, New York: Humana Press, p. 7-37.

Alves, R. R. N., Silva, J. S., da Silva Chaves, L., \& Albuquerque, U. P. (2017). Ethnozoology and animal conservation. In: Alves R.N., Albuquerque, U.P., editors. Ethnozoology, Animals in our Lives. Academic Press, p. 481-496.

Bertonio, L. [1612] (2006). Vocabulario de la Lengua Aymara (transcripción del texto original de 1612). Arequipa: Ediciones El Lector.

Chávez, E. (2019). El Chollonco Mollendino. Diario Prensa Regional. Disponible en: https://prensaregional.pe/el-chollonco-mollendino/

Daut, E. F., Brightsmith, D. J., Mendoza, A. P., Puhakkac, L., \& Peterson, M. J. (2015). Illegal domestic bird trade and the role of export quotas in Peru. Journal for Nature Conservation, 27, 44-53. DOI: 10.1016/j.jnc.2015.06.005

De la Peña, M. R. (2011). Diccionario de nombres vulgares de las aves de Argentina. Serie Naturaleza, Conservación y Sociedad $N^{\circ} 1$. Santa Fe, Argentina: Ediciones Biológica.

Farias, G. B., \& Alves, Â. G. C. (2007). Aspectos históricos e conceituais da etnoornitologia. Biotemas, 20(1), 91-100.

Gosler, A. (2010). The Broader Significance of Ethno-ornithology. In: Tidemann S., Gosler, A., editors. Ethno-ornitology. Birds, Indigenous Peoples, Culture and Society. London: Earthscan, p. 31-39.

Hughes, R. A. (1991). Las Aves de la Provincia de Islay. Boletín de Lima, 75, 47-54.

INEI. (2020). Perú: Proyecciones de Población, Según Departamento, Provincia y Distrito, 2018-2020. Boletín especial del Instituto Nacional de Estadística e Informática, 25, 1-110. 
INRENA. (1996). Anuario Estadístico de Exportaciones de Flora y Fauna Silvestres Año 1995. Lima: Instituto Nacional de Recursos Naturales-Dirección General de Áreas Protegidas y Fauna Silvestre. Ministerio de Agricultura.

Luque, C., Villegas, L., Caballero, K., \& Samata, D. (2019). Aves de Arequipa, Lista anotada. Arequipa: Instituto de Investigación y Gestión Ambiental, Universidad Nacional de San Agustín. Disponible en: https://www.researchgate.net/publication/335777841_Aves_de_Arequipa_Lista_anotada

Ministerio de Agricultura. (1981). Anuario de Estadística Forestal y de Fauna Silvestre 1981. Lima: Dirección General Forestal y de Fauna.

Ministerio de Agricultura. (1991). Perú Forestal en Números. Lima: Dirección General Forestal y de Fauna.

Ortiz, C. A. (2010). Aves silvestres comercializadas en el mercado de productores El Palomar-Arequipa. Boletín de Lima, 32(159), 136-140.

Ortiz, C. A. (2017). Primera Descripción del Nido del Chirigüe de Raimondi (Sicalis raimondii) en Arequipa, Perú. Ornitología Neotropical, 28, 219-221.

Pulido, V. (1998). Vocabulario de los nombres comunes de la fauna silvestre del Perú. Lima: D. Velarde ed.

Rundel, P. W., Dillon, M. O., Palma, B., Mooney, H. A., Gulmon, S. L., \& Ehleringer, J. R. (1991). The Phytogeography and Ecology of the Coastal Atacama and Peruvian Deserts. Aliso, 13(1), 1-50.

Sault, N. (2010). Bird Messengers for All Seasons: Landscapes of Knowledge among the Bribri of Costa Rica. In: Tidemann S., Gosler A., editors. Ethno-ornitology. Birds, Indigenous Peoples, Culture and Society. London: Earthscan, p. 291-300.

Schulenberg, T. S., Stotz, D. F., Lane, D. L., O'neill, J. P., \& Parker III, T. A. (2007). Birds of Peru: revised and updated edition. Princeton, N.J.: Princeton University Press.

SERNANP. (2016). Guía informativa. Listado, Clasificación y Abundancia de Aves. Santuario Nacional Lagunas de Mejía. Lima: Servicio nacional de áreas naturales protegidas por el Estado. 